\title{
Etude préliminaire de la malacofaune de deux hydrosystèmes interconnectés dans une zone humide
}
A. Blanc 1
C. Gérard ${ }^{1}$

Mots-clés : Mollusques, communautés, zones humides, eau stagnante/courante.

Dans le cadre de la gestion préventive des zones humides, une analyse descriptive des communautés malacologiques a été réalisée pendant la saison sèche dans deux hydrosystèmes interconnectés, lentique et lotique, d'une zone humide. Au total, 2182 Mollusques ont été collectés, appartenant à 6 familles de Gastéropodes (Hydrobiidae, Planorbidae, Physidae, Lymnaeidae, Ancylidae, Acroloxidae) et une famille de Bivalves (Sphaeriidae). Malgré une connexion entre les deux hydrosystèmes, les communautés sont très différentes et la richesse spécifique est nettement supérieure dans le milieu lentique vs lotique. Les Bivalves, très rares dans le cours d'eau, représentent la moitié des Mollusques dans le bras mort. Les Gastéropodes du bras mort sont exclusivement des Pulmonés (8 espèces) alors que ceux du cours d'eau appartiennent pour $99,5 \%$ à une seule espèce de Prosobranche, Potamopyrgus antipodarum. Les exigences écologiques des Mollusques sont discutées en relation avec les caractéristiques des deux milieux, mais ne suffisent pas à expliquer l'extrême rareté d'espèces communes.

\section{Description of malacological communities in two interconnected hydrosystems in wetlands}

Keywords : Molluscs, communities, wetlands, stagnant/running waters.

In context of the preventive management of wetlands, a descriptive analysis of malacological communities was undertaken during the dry season in two interconnected freshwater ecosystems, a pond and a stream, in a wetland. A total of 2,182 Molluscs was collected, belonging to 6 families of Gastropods (Hydrobiidae, Planorbidae, Physidae, Lymnaeidae, Ancylidae, Acroloxidae) and one family of Bivalves (Sphaeriidae). Although the hydrosystems are interconnected, the communities are very different and species richness is greater in stagnant vs running water. Bivalves, very rare in the stream, represent half the Molluscs in the pond. All Gastropods in stagnant water are Pulmonates (8 species), and $99.5 \%$ of Gastropods in the stream belong to a single species of Prosobranches, Potamopyrgus antipodarum. Ecological constraints of Molluscs are discussed in relation to environmental characteristics, but don't explain the extreme rarity of species living in the two hydrosystems.

\section{Introduction}

Les-zones humides sont connues pour jouer un rôle capital dans la dépollution, en particulier via les processus de dénitrification (Haycock et al. 1997). Les Mollusques dulcicoles, en raison de leur rôle-clé dans le fonctionnement des écosystèmes et de leur sensibilité aux variations des conditions environnementales,

\footnotetext{
1. UMR CNRS 6553 «Ecobio», Université de Rennes 1, Avenue du Général Leclerc F-35042 Rennes cedex.

E-mail : alexia.blanc@univ-rennes1.fr ; claudia.gerard@univrennesl.fr
}

peuvent constituer des bio-indicateurs potentiels de pollution (Mouthon \& Charvet 1999). Dans le cadre de la gestion préventive des zones humides menacées par les excès de l'agriculture intensive (programme CNRS Environnement, Vie et Société), nous avons réalisé un état des lieux de la malacofaune dans le compartiment aquatique d'une zone humide en aval de cultures maraîchères.

\section{Matériel et méthodes}

Située à Pleine-Fougères dans la Baie du MontSaint-Michel (France, latitude Nord $48^{\circ} 24^{\prime}$, longitude Ouest $1^{\circ} 45^{\prime}$ ), la zone humide étudiée est typique des fonds de vallons bretons sur schiste. Le compartiment 
aquatique y est constitué par Le Petit Hermitage, ruisseau qui rejoint le Couesnon en aval, et par le bras mort, zone de rétention au centre de la plaine alluviale et vestige du cours d'eau initial (Fig. 1). Les deux hydrosystèmes communiquent largement en période de crue (Novembre-Février), période en dehors de laquelle des pertes hydriques latérales sont démontrées du cours d'eau vers le bras mort (Lefebvre 2000). Chacun des quatre prélèvements réalisés dans chaque site pendant l'été 2000 est effectué à l'aide d'un filet-troubleau (maille de nylon : $1 \mathrm{~mm}$, ouverture carrée : 0,5 x 0,5 $\mathrm{m})$, par deux personnes pendant 5 minutes dans une bande littorale de $40 \mathrm{~m}^{2}$ de surface et de profondeur maximale de $0,5 \mathrm{~m}$. Le contenu des troubleaux est examiné au laboratoire. Aucun individu n'est remis à l'eau entre chaque prélèvement.
Tous les Mollusques collectés (2182 individus) sont identifiés au rang d'espèce, à l'exception de Pisidium sp. qui présente une grande proportion de juvéniles.

\section{Résultats et discussion}

Malgré l'interconnexion des 2 hydrosystèmes, leur proximité $(50 \mathrm{~m})$, et donc, les possibilités de colonisation concomitantes par les mêmes espèces de Mollusques, les communautés malacologiques présentent des structures très différentes (Tableau 1). Dans le ruisseau, 97,7\% des Mollusques sont des Gastéropodes Prosobranches : Potamopyrgus antipodarum, alors que dans le bras mort, la communauté est composée pour moitié par des Bivalves et pour moitié par des Gastéropodes Pulmonés (8 espèces). La richesse spécifique,

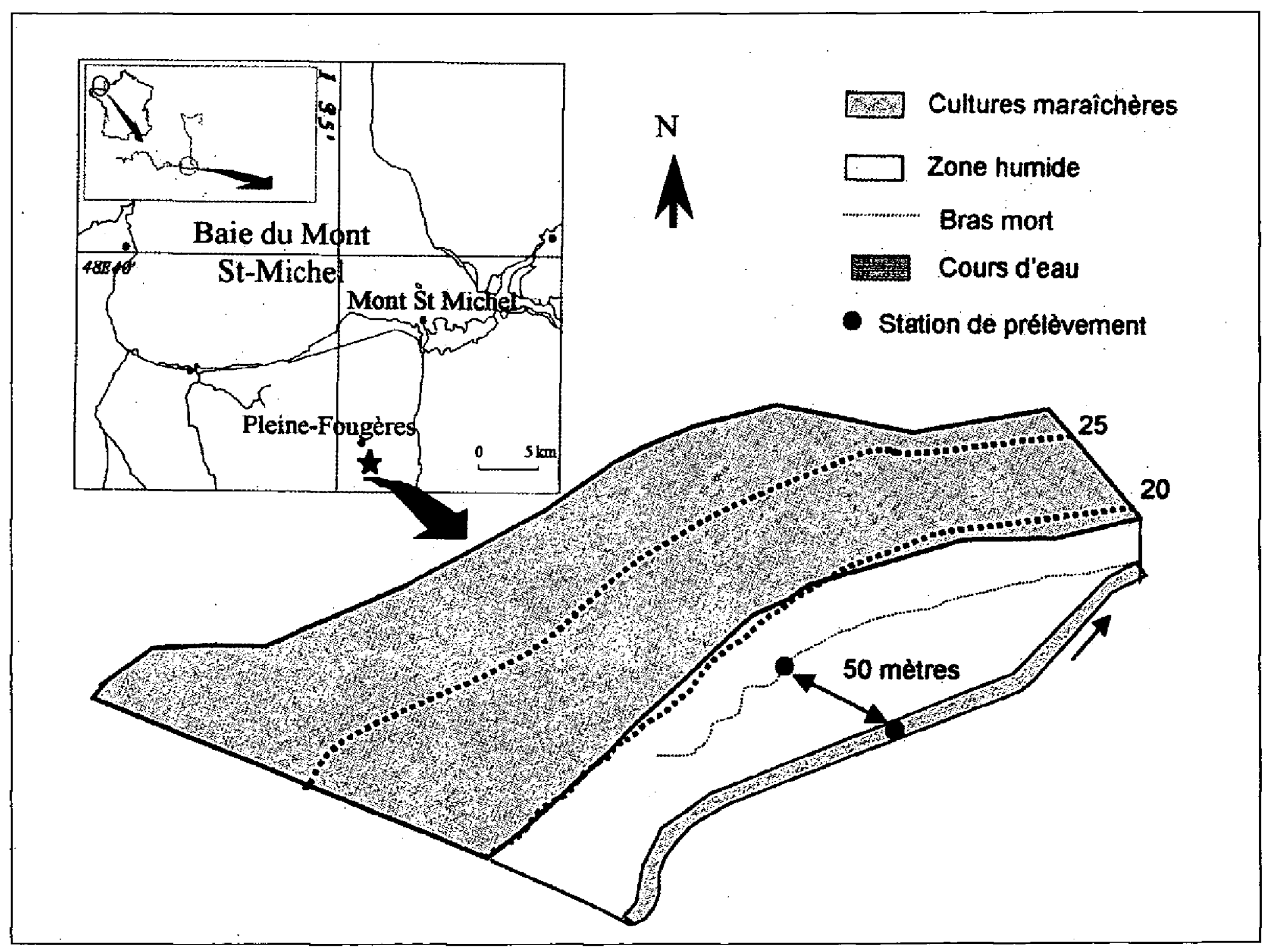

Fig. 1. Site de Pleine-Fougères (Baie du Mont-Saint-Michel, France) (d'après Clément 2001).

Fig. 1. Study-site in Pleine-Fougères (Mont-Saint-Michel basin, France) (in Clément 2001). 
Tableau 1. Communautés de Mollusques dans deux hydrosystèmes de la zone humide de Pleine-Fougères (été 2000).

Table 1. Malacological communities of two hydrosystems in the wetland of Pleine-Fougères (summer 2000).

\begin{tabular}{|c|c|c|c|c|}
\hline & \multicolumn{2}{|c|}{ Milieu lentique (bras mort) } & \multicolumn{2}{|c|}{ Milieu lotique (cours d'eau) } \\
\hline & Effectif & Fréquence \% & Effectif & Frequence \% \\
\hline \multicolumn{5}{|l|}{ Gastéropodes } \\
\hline Potamopyrgus antipodarum (Smith) & & & 638 & 97.70 \\
\hline Planorbis planorbis (Linné) & 463 & 30.28 & & \\
\hline Aplexa hypnorum (L.) & 28 & 1.83 & & \\
\hline Physa fontinalis (Linné) & 3 & 0.20 & & \\
\hline Physella heterostropha (Say) & 250 & 16.35 & 1 & 0.15 \\
\hline Physella acuta (Draparnaud) & 8 & 0.52 & & \\
\hline Stagnicola glaber (O.F. Müller) & 2 & 0.13 & & \\
\hline Radix peregra (O.F. Müller) & 7 & 0.46 & & \\
\hline Radix ovata (Draparnaud) & 1 & 0.07 & & \\
\hline Acroloxus lacustris (L.) & & & 1 & 0.15 \\
\hline Ancylus fluviatilis (O.F. Müller) & & & 1 & 0.15 \\
\hline \multicolumn{5}{|l|}{ Bivalves } \\
\hline Pisidium sp. (Pfeiffer) & 767 & 50.16 & 11 & 1.68 \\
\hline Sphaerium comeum (L.) & & & 1 & 0.15 \\
\hline Total Gastéropodes & 762 & 49.84 & 641 & 98.16 \\
\hline Total Bivalves & 767 & 50.16 & 12 & 1.84 \\
\hline Total Mollusques & 1529 & $100 \%$ & 653 & $100 \%$ \\
\hline
\end{tabular}

est beaucoup plus élevée dans les eaux stagnantes (bras mort) vs courantes (ruisseau) en accord avec Pip (1987). Les différences écologiques des deux hydrosystèmes expliquent en grande partie les différences de structure des communautés. D'après les nombreux travaux sur l'écologie des Mollusques (pour revues : Boycott 1936, Okland 1990), le ruisseau, qui présente un courant fort associé à un substrat de type gravier et une végétation aquatique pauvre, constitue un biotope beaucoup moins favorable aux Mollusques que le bras mort, eau stagnante à tendance eutrophe, caractérisé par un substrat sablo-vaseux et une végétation aquatique développée.

Cependant, ces différences écologiques ne suffisent pas à expliquer pourquoi il n'existe pas plus d'espèces communes aux deux communautés, compte tenu de la communication entre les deux hydrosystèmes. En effet, si l'on excepte Pisidium sp. (rang spécifique non déterminé), une seule des 12 espèces de Mollusques récoltées, Physella heterostropha, a été rencontrée dans les 2 milieux : 250 individus dans le bras mort ( $2^{\text {ème }}$ rang d'abondance) et un seul individu dans le cours d'eau. D'autre part, $P$. antipodarum, espèce invasive (Ponder 1988), est absente du bras mort, alors qu'elle domine largement la communauté malacologique du cours d'eau, et qu'elle partage avec Planorbis planorbis, l'espèce dominante du bras mort, le même régime alimentaire (Reavell 1980). Présente dans les eaux saumâtres et dulcicoles de la Baie du Mont-Saint-Michel (Costil et al. 2001), P. antipodarum peut vivre en eau stagnänte en Norvège, où au moins 5 espèces lui sont en moyenne associées (Okland 1990). D'autre part, elle coexiste dans d'autres milieux lotiques de la Baie du Mont-Saint-Michel avec plusieurs espèces de Planorbidae, Physidae et Lymnaeidae présentes dans le bras mort à Pleine- 
Fougères (résultats non publiés), et considérées pour la plupart comme tolérantes.

Dans la mesure où les eaux de ruissellement du bassin versant, qui deviennent stagnantes dans le bras mort ou alimentent le cours d'eau, sont potentiellement vectrices de pollution, la distribution des espèces de Mollusques dans le compartiment aquatique de la zone humide peut être liée à leur sensibilité vs tolérance à la qualité de l'eau. L'analyse physicochimique de l'eau et les études menées sur le devenir des polluants dans la zone réceptrice du bassin-versant de Pleine-Fougères permettront d'expliquer la structure des peuplements malacologiques dans les deux hydrosystèmes.

\section{Remerciements}

Nous tenons à remercier Maryline Steinhard pour son assistance technique. Cette étude a bénéficié d'un financement du C.N.R.S. dans le cadre du Programme Environnement, Vie et Société.

\section{Travaux cités}

Boycott A.E. 1936. - The habitats of freshwater Mollusca in Britain. J. Anim. Ecol., 5 : 116-186.
Clément J.C., 2001. - Les zones humides de fonds de vallée et la régulation des pollutions azotées diffuses. Thèse de l'Université de Rennes $1: 183 \mathrm{p}$.

Costil K., Dussart G.B.J. \& Daguzan J. 2001. - Biodiversity of aquatic gastropods in the Mont-Saint-Michel basin (France) in relation to salinity and drying of habitats. Biodivers. Conserv., 10 : $1-18$.

Haycock N.E., Burt T.P., Goulding K.W.T. \& Pinay G. 1997. - Buffer zones : their processes and potential in water protection. Quest Environmental, Harpenden, UK : $320 \mathrm{p}$.

Lefebure S. 2000. - Qualité des cours d'eau en bassins versants agricoles : capacités auto-épuratrices de l'interface eau-sédiments dans differents contextes d'anthropisation. DEA Environnement Temps, Espaces, Sociétés (Muséum National d'Histoire Naturelle) : $54 \mathrm{p}$.

Mouthon J. \& Charvet S. 1999. - Compared sensitivity of species, genera and families of Molluses to biodegradable pollution. Ann. Limnol., 35 (1) : 31-39.

Okland J. 1990. — Lakes and snails. Environment and Gastropoda in 1,500 Norvegian lakes, ponds and rivers. Universal Book Services, Oegstgeest : $516 \mathrm{p}$.

Pip E. 1987. - Species richness of freshwater gastropod communities in central North America. J. Moll. Stud., 53 : 163-170.

Ponder W.F. 1988. - Potamopyrgus antipodarum - a molluscan coloniser of Europe and Australia. J. Moll. Stud., 54 : 271-285.

Reavell P.E. 1980. - A study of the diets of some british freshwater Gastropods. J. Conc., $30: 253-271$. 